\title{
MaMed 02: Nutzung medizinischer Zeitschriften
}

Erfolgreich sind wir nur, wo wir nützen ...

Emil Oesch

Soeben sind die Resultate der Publikumsbefragung «MaMed 02» erschienen, auf die wir beim Schweizerischen Ärzteverlag EMH gespannt gewartet haben. Würden sie uns Aufschluss darüber geben, wie sich unser neues Konzept, aus der früheren SÄZ und der früheren SMW drei neue Zeitschriften zu machen, bewährt hat?

Natürlich haben wir auf gute Resultate gehofft, aber sie haben unsere Erwartungen weit übertroffen, und darüber freuen wir uns enorm.

Warum sollte Sie dies alles interessieren? Und was ist diese MaMed überhaupt?

MaMed ist mit gewissen Einschränkungen eine vergleichende Studie der Nutzung und Akzeptanz medizinischer Zeitschriften. Den Hintergrund bildet dabei das Bedürfnis der Firmen, die in medizinischen Zeitschriften Inserate schalten, Entscheidungskriterien in die Hand zu bekommen, wo sich Werbung lohnt. Um solche Kriterien auszuarbeiten, haben die wesentlichen Medizinverlage der Schweiz MaMed beim renommierten GfS-Forschungsinstitut in Auftrag gegeben. Die Einschränkungen liegen damit nicht in der Qualität der Studie (sie ist durch das GfS garantiert), sondern in der Studienanlage: Es geht um eine Befragung innerhalb einer Grundgesamtheit von 7000 Kolleginnen und Kollegen Allgemeinmedizinern, Internisten, Gynäkologen und Psychiatern mit eigener Praxistätigkeit. Es wurde also nur ein Segment der Ärzteschaft befragt; alle im Spital tätigen Ärztinnen und Ärzte waren nicht eingeschlossen. Es ist nun nicht so, dass wir in den Redaktionen uns nicht für sämtliche Leserinnen und Leser interessieren, aber MaMed ist keine redaktionelle Studie; die Stichprobe wurde in Absprache mit Vertretern der vips (Vereinigung der Importeure pharmazeutischer Spezialitäten) festgelegt. Die Publikation medizinischer Fachzeitschriften wäre heute ohne Einnahmen aus dem Inseratemarkt nicht möglich - es sei denn, Sie wären als Leser bereit, mehrere hundert Franken pro Abonnement zu bezahlen. Studien wie die MaMed können dazu beitragen, dass wir Ihnen weiterhin die für Sie wichtigen Zeitschriften zu äusserst günstigen Bedingungen zustellen können.

Nun, wie wichtig sind Ihnen denn die Zeitschriften, die wir allen FMH-Mitgliedern jede Woche zusenden?

Es hat sich gezeigt, dass die Schweizerische Ärztezeitung als das wichtigste gesundheitspolitische Informationsmedium praktisch von allen befragten Kolleginnen und Kollegen genutzt wird: Der «weiteste Leserkreis» (WLK) der SÄZ beträgt 93\%! Aus- serordentlich freut uns, dass wir auch im Fortbildungsbereich Spitzenreiter sind: Das Swiss Medical Forum liegt auf Rang 2 mit einem WLK von 76\%. Diese Zahlen sprechen für sich.

Der Schlussbericht zur MaMed 02 kann auf der Website http://www.gfs.ch/publikationen/medzeit eingesehen werden.

Für das grosse Interesse der niedergelassenen Ärzteschaft am wissenschaftlichen Geschehen in der Schweiz spricht die Treue zur alten SchwiWo, jetzt Swiss Medical Weekly. Trotz des gewagten Wechsels zur ausschliesslichen Publikation von englischsprachigen Originalarbeiten sind die Abonnementszahlen weit höher geblieben, als kritische Stimmen uns prophezeien wollten.

Das Konzept, Ihnen Information in drei spezifisch konzipierten Gefässen anzubieten, scheint also aufzugehen. Für uns im Verlag und in den Redaktionen ist dies ein enormer Ansporn. Nächste Schritte sind bereits in der Pipeline. Wir werden die Interaktivität ausbauen und die Angebote unserer Zeitschriften-Websites dazu einsetzen. Denn auch das hat die MaMed gezeigt: Ärztinnen und Ärzte nutzen das Internet zur gezielten Informationssuche. Dies bestätigt auch die Erfahrung aus unseren Website-Statistiken. Alle EMH-Zeitschriften haben eine eigene Website mit Archiv und Volltextsuche, deren Zugriffszahlen bereits hoch sind und weiter steil ansteigen.

«Wenn Du trinkst, gedenke der Quelle», sagt ein chinesisches Sprichwort. Die Quelle unseres Erfolges sind die Autorinnen und Autoren, die für unsere Zeitschriften schreiben, die Reviewer, die Beiträge gegenlesen oder Verbesserungsvorschläge einbringen, und alle die vielen Leserinnen und Leser, die uns ihr Feedback zukommen lassen. Gerade beim Swiss Medical Forum ist die Bereitschaft der angefragten Autorinnen und Autoren zur Kooperation überwältigend, und das ist nicht selbstverständlich. Um so mehr möchten wir allen, die mit ihrer Mitarbeit unsere Zeitschriften erst möglich machen, an dieser Stelle herzlich danken!

Wir haben oft betont, dass EMH sich als der Verlag der Ärztinnen und Ärzte versteht, als Ihr Verlag, mit der Aufgabe, für Sie jene Produkte zu publizieren, die Sie von uns erwarten. Die MaMed 02 zeigt uns, dass wir auf dem richtigen Weg sind. Mehr nicht, aber auch nicht weniger. Wir werden in dieser Richtung weiterarbeiten, aber auch Ihre Kritiken und Anregungen weiterhin sehr ernst nehmen und jederzeit dafür offenbleiben, etwas zu verändern, das der Qualität unserer Produkte dienen kann.

Für den Verwaltungsrat EMH: Natalie Marty, Ludwig T. Heuss 


\section{MaMed 02: De l'usage des journaux médicaux}

Nous ne rencontrons le succès que là

où nous sommes utiles ...

Emil Oesch

Les résultats de l'enquête «MaMed 02», que nous attendions avec impatience aux Editions médicales suisses, viennent de sortir. Saurons-nous enfin quel écho reçoit notre nouveau concept (trois publications à la place de deux - à savoir les anciennes versions du Bulletin des médecins suisses et du Journal Suisse de Médecine)?

Nous espérions naturellement des résultats encourageants, mais vos réponses ont largement dépassé notre attente, ce qui à tout lieu de nous réjouir.

En quoi cela doit-il vous intéresser? En fait, qu'est-ce donc que l'enquête MaMed?

MaMed est une étude comparative sur l'usage et l'acceptance des journaux médicaux. A l'origine de cette étude se trouvent les besoins des entreprises qui mettent des annonces dans les journaux et qui cherchent à établir des critères de décision quant à la rentabilité des annonces (quelle annonce pour quel journal). Pour définir de tels critères, les principaux éditeurs médicaux de la Suisse ont chargé le célèbre institut de recherche GfS de mener l'enquête. Les restrictions à cette étude n'ont rien à voir avec la qualité, cette dernière étant garantie par GfS, mais avec la population de l'enquête qui ne comprend pas la totalité des médecins. Elle couvre un groupe de plus de 7000 collègues présentant une certaine similarité - médecins généralistes, internistes, gynécologues et psychiatres exerçant en cabinet; les médecins exerçant en hôpital n'étant donc pas pris en compte. Il ne faut pas voir dans ce choix d'un segment de la profession médicale un désintérêt de la rédaction pour une partie de ses lecteurs, mais simplement le fait que MaMed n'est pas une étude lancée par la rédaction elle-même. L'échantillon était fixé en accord avec des représentants de la vips (Association des importeurs de spécialités pharmaceutiques). La publication de journaux médicaux spécialisés serait financièrement impossible sans le marché des annonces (à moins que vous ne soyez prêts, chers lecteurs, à payer un abonnement de plusieurs centaines de francs ...). Et nous sommes donc redevables à des études comme MaMed de nous permettre de continuer à vous adresser ces revues médicales importantes pour votre information à des conditions très avantageuses.

$\mathrm{Au}$ fait, à quel point les journaux que nous adressons chaque semaine aux membres de la FMH vous intéressent-ils?

Il s'avère que le Bulletin des médecins suisses est utilisé comme média d'information essentiel sur les questions de politique de santé, par pratiquement tous les collègues interrogés. Le cercle des lecteurs «le plus large» du BMS est même de $93 \%$ ! Nous nous réjouissons également de notre position de tête dans le domaine de la formation continue, avec Swiss Medical Forum (en deuxième place, avec 76\% de lecteurs). Ces chiffres sont très évocateurs.

Le rapport final de MaMed 02 (en allemand) peut être consulté sur la site web http://www.gfs.ch/ publikationen/medzeit.

La fidélité à l'hebdomadaire devenu maintenant le Swiss Medical Weekly témoigne de l'intérêt des médecins installés pour l'actualité scientifique en Suisse. Malgré le changement audacieux en une publication exclusivement en anglais, le nombre des abonnés est resté bien plus élevé que ne nous le prédisaient les critiques et mauvais augures.

Le concept de trois publications ayant chacune son domaine spécifique semble donc recevoir l'assentiment du public, ce qui constitue un grand encouragement pour les membres de la rédaction. Les étapes suivantes sont déjà en préparation. Nous voulons développer l'interactivité et élargir à cette fin les possibilités des journaux publiés sur notre site Internet. Car c'est aussi un résultat de l'enquête MaMed: les médecins recourent de plus en plus à Internet pour la recherche ciblée d'informations. C'est d'ailleurs ce que confirment nos propres statistiques. Toutes les publications des EMH ont leur propre site, comportant des archives et une recherche par texte. Le nombre de visites sur ces sites est déjà très élevé et ne manquera pas d'augmenter.

"Quand tu bois, pense à la source», dit un proverbe chinois. La source de notre succès, ce sont les auteurs qui écrivent pour nos revues, les reviewers qui relisent les manuscrits ou encore proposent des améliorations, et ce sont aussi nos lecteurs, qui nous adressent leur feed-back. La coopération des auteurs - ceux sollicités pour Swiss Medical Forum tout particulièrement - est absolument remarquable. Cela n'a rien d'évident, et nous tenons d'autant plus à remercier sincèrement ici tous les collaborateurs de nos journaux!

Nous avons souvent souligné que les EMH se comprennent comme l'éditeur des médecins, comme votre éditeur, se fixant pour tâche de publier ce que vous attendez de nous. L'enquête MaMed 02 nous montre - ni plus, ni moins - que nous sommes sur la bonne voie. Nous continuerons à travailler dans ce sens, en accueillant vos critiques et vos suggestions, et en restant ouverts à tout changement permettant d'améliorer la qualité de nos produits.

Pour le Conseil d'administration EMH: Natalie Marty, Ludwig T. Heuss 